\title{
Promotion of Osseointegration between Implant and Bone Interface by Titanium Alloy Porous Scaffolds Prepared by 3D Printing
}

Yuhao Zheng ${ }^{a, b, c}$, Qing Han ${ }^{d}$, Jincheng Wang ${ }^{d}$, Dongdong Li ${ }^{e, ~ *, ~ Z h i m i n g ~ S o n g ~}{ }^{a}$, , and Jihong $Y u^{b, c, *}$

a Department of Sports Medicine, First Hospital of Jilin University, Changchun 130021, P. R. China

${ }^{b}$ State Key Laboratory of Inorganic Synthesis and Preparative Chemistry, College of Chemistry, Jilin University, Changchun 130012, P. R. China

${ }^{c}$ International Center of Future Science, Jilin University, Changchun 130012, P. R. China

${ }^{d}$ Department of Joint Surgery, Orthopedic Medical Center, Second Hospital of Jilin University, Changchun 130000, P. R. China

${ }^{e}$ Key Laboratory of Automobile Materials of MOE, Department of Materials Science and Engineering, Jilin University, Changchun 130012, P. R. China

*Dongdong Li E-mail: lidongdong@jlu.edu.cn

* Zhiming Song E-mail: szm3210@163.com

*Jihong Yu E-mail: jihong@jlu.edu.cn 


\section{Materials and Methods}

\section{Material of the Scaffolds}

Ti-6Al-4V Powder morphology was evaluated using a scanning electron microscope. As shown in Figure S1A, the diameter of the powder was between 12 and $24 \mu \mathrm{m}$, and the diameter of the powder was small, with an average diameter of 17.84 $\mu \mathrm{m}$. The particle size distribution is shown in Figure S1B.

\section{Compression tests of Scaffolds}

Compression tests were conducted to determine the mechanical properties of the Ti6Al4V alloy scaffolds with different pore sizes. The tests were performed using an electronic universal testing machine (CSS-44100, Changchun Testing Machine Research Institute, Changchun, P. R. China). The maximum load of the instrument was $100 \mathrm{kN}$, and the injection volume during the experiment was $0.5 \mathrm{~mm} \mathrm{~min}^{-1}$.

As shown in Figure S2, the principle of the compressive strength was as follows: P400 > P320 > P600 > P800. When the compression displacement of P400 reaches $0.392 \mathrm{~mm}$, the compressive stress reaches a peak value of $5.04 \mathrm{kN}$.

\section{Cytotoxicity of Scaffolds}

Methylthiazol tetrazolium assay was carried out to evaluate cell proliferation. First, each group of high-temperature sterilized scaffolds was co-cultured with $100 \mu \mathrm{l}$ BMSCs in 96-well plates for 24 and 48 hours. The concentration of BMSCs was $5 \times$ $10^{4}$ cells per milliliter and the Control group was $100 \mu \mathrm{BMSCs}$ without scaffold in the well. Second, removed all scaffolds from 96-well plates and MTT solution was prepared by dissolving MTT in PBS. After MTT solution was added to each sample, 
the plates were incubated at $37^{\circ} \mathrm{C}$ for $4 \mathrm{~h}$ to form formazan, which was then dissolved using dimethyl sulfoxide (DMSO). The optical density (OD) was measured using a spectrophotometer at a wavelength of $490 \mathrm{~nm}$. At least five samples were analyzed for each group and the cell viability was expressed by the formula below.

$$
\lambda=\left(\mathrm{A}_{\text {sample }} / \mathrm{A}_{\text {control }}\right) \times 100 \%
$$

Where $\lambda$ represents relative cell viability. $A_{\text {sample }}$ represents OD value of samples. $\mathrm{A}_{\text {control }}$ represents OD value of Control group.

As shown in Figure S3, the scaffolds in each group had no toxic effect on cell proliferation $(P>0.05)$ after co-culture with BMSCs for 24 hours or 48 hours. This indicated that no toxic substances were introduced during the preparation and pretreatment of the scaffolds.
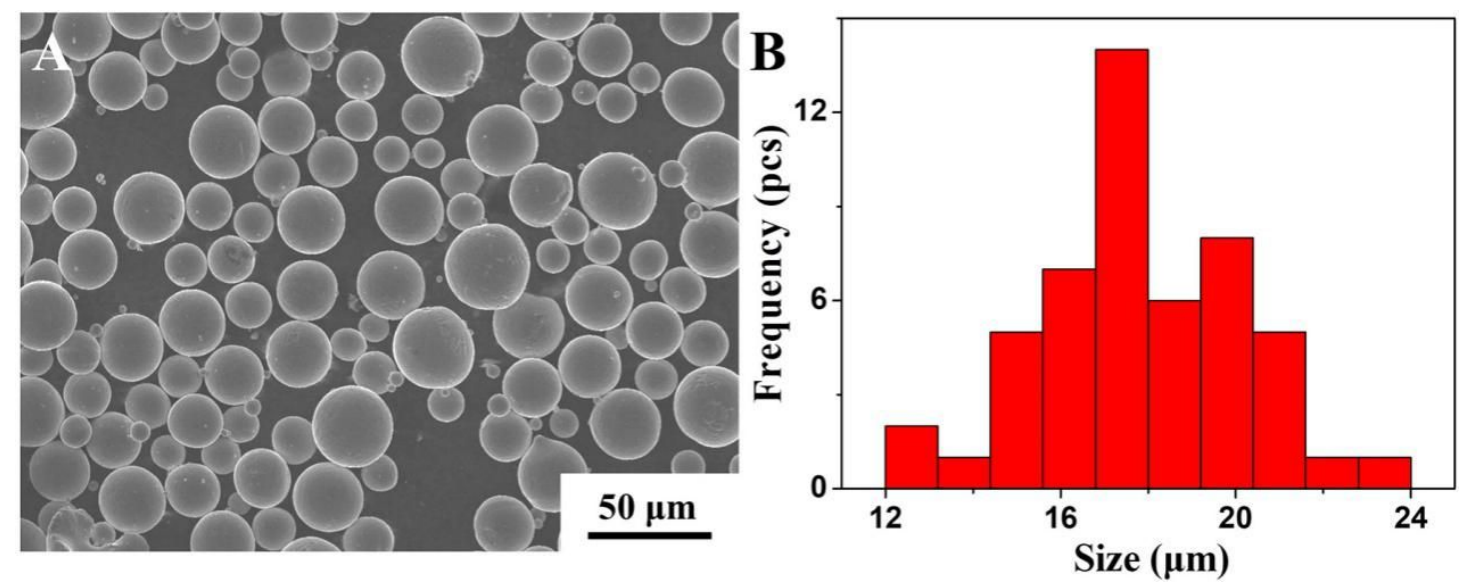

Figure S1. Morphology and scale of the Ti-6Al-4V powder. (A) Scanning electron microscope image and (B) size distributions of the Ti-6Al-4V powder $(n=50)$. 


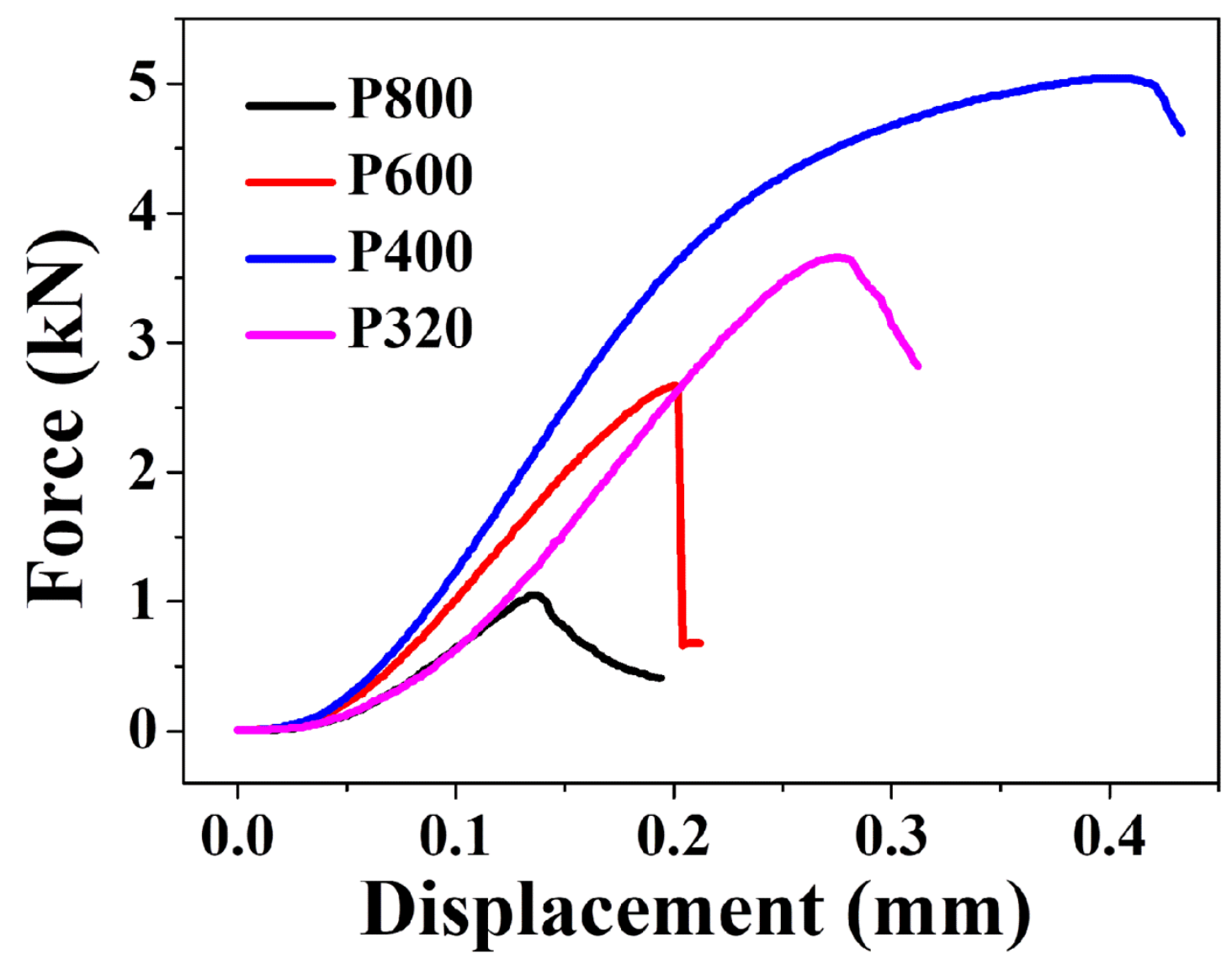

Figure S2. Compressive load-displacement curves of scaffolds with different pore sizes.
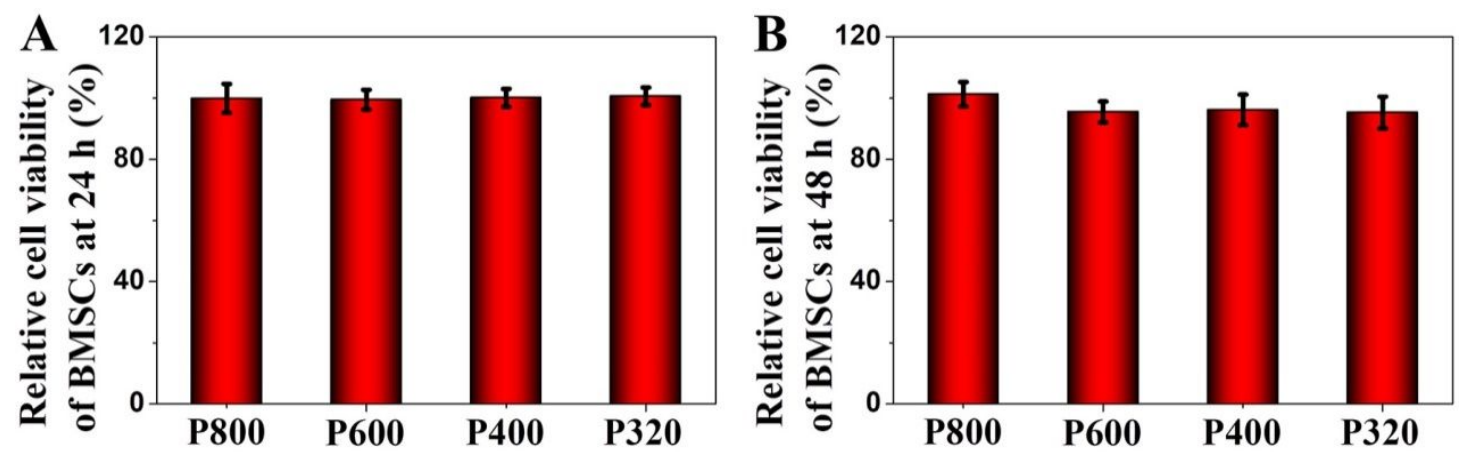

Figure S3. In vitro relative cell viability of $\mathrm{P} 800, \mathrm{P} 600, \mathrm{P} 400$ and $\mathrm{P} 320$ after incubation with BMSCs for 24 (A) and $48 \mathrm{~h}$ (B). Each value represents the mean \pm $\mathrm{SD}(\mathrm{n}=5)$. 